\title{
Secondary renal neoplasms
}

\author{
J. E. Bailey, M. A. Roubidoux, N. R. Dunnick \\ Department of Radiology, University of Michigan Medical Center, 1500 East Medical Center Drive, Ann Arbor, MI 48109-0030, USA
}

The widespread use of computed tomography (CT) and sonography has led to the increased detection of renal masses, many of which are small and asymptomatic. In general, solid renal masses are treated as renal adenocarcinomas until proven otherwise [1]. This treatment often results in nephrectomy, some of which are unnecessary.

A renal metastasis can be suspected when there is an underlying primary tumor. Although the imaging characteristics are not pathognomonic, the imaging features in the appropriate clinical setting may lead to the correct diagnosis and the most appropriate management.

\section{Renal metastasis}

A renal metastasis is the most common malignant neoplasm of the kidney found at autopsy [2]. In their review of 1000 consecutive postmortem examinations of patients with malignant neoplasms of epithelial origin, Abrams et al. found metastases to the kidneys in 126 (12.6\%) cases [3]. Klinger reported 118 cases of renal metastases (including lymphomas) in 5000 autopsies (2.4\%) [4]. When lymphoma was excluded, 73 patients had a renal metastasis at autopsy, representing $1.5 \%$ of the general population. Wagle et al. reported an incidence of nonlymphoma renal metastases of $1.8 \%$ from a series of 4413 autopsies [5]. These large autopsy series found that the most common primary tumors to metastasize to the kidneys are lung (19.8-23.3\%), breast (12.3\%), and gastric carcinomas (11.1-15.1\%). With the declining incidence of gastric carcinoma and rising incidence of melanoma, it is likely that melanoma is the third most frequent tumor to metastasize to the kidney.

Although metastases to the kidney are common, they are seldom symptomatic and they do not produce

Correspondence to: N. R. Dunnick laboratory changes such as hematuria or azotemia. In the series reported by Wagle et al. [5], none of the patients had gross hematuria and only $31 \%$ had microscopic hematuria. Hematuria was reported even less frequently by Klinger [4] (19\%) and Ollson et al. [6] (12\%). Renal failure is even less common; it was present at the time of death in only $5 \%$ of the patients reported by Wagle et al. Thus, renal metastases are rarely suspected on clinical grounds. With more frequent use of CT, ultrasound, and magnetic resonance (MR), metastases are more likely to be detected as incidental findings.

The pathology of renal metastases may explain their clinical silence. Metastatic deposits are found at autopsy as multiple cortical nodules via hematogenous seeding from entrapment of tumor cells in the glomerular capillary tuft [6]. Involvement of the urothelial mucosa is uncommon, which accounts for the low incidence of hematuria, even with large lesions [4].

Patients with renal metastases studied at autopsy tend to have widely disseminated malignant disease. Of 60 patients with renal metastases from lung carcinoma studied by Olsson et al., only 3\% had no other sites of involvement [6]. Combining three autopsy studies and excluding lymphomas, $54 \%$ of the patients with renal metastases had bilateral lesions [4-6].

\section{Imaging of renal metastasis}

Excretory urography (IVP) has a low sensitivity for detecting metastatic tumors. In two recent series, the falsenegative rate was high because urography was able to detect only four of nine and none of six renal metastases $[7,8]$. Lesions found by urography are large and exophytic, causing a contour abnormality (Fig. 1) or distortion of collecting system [7]. Small lesions are poorly seen, even with tomography, because they rarely alter the renal contour or distort the collecting system. Renal metastases rarely manifest as intraluminal filling defects 


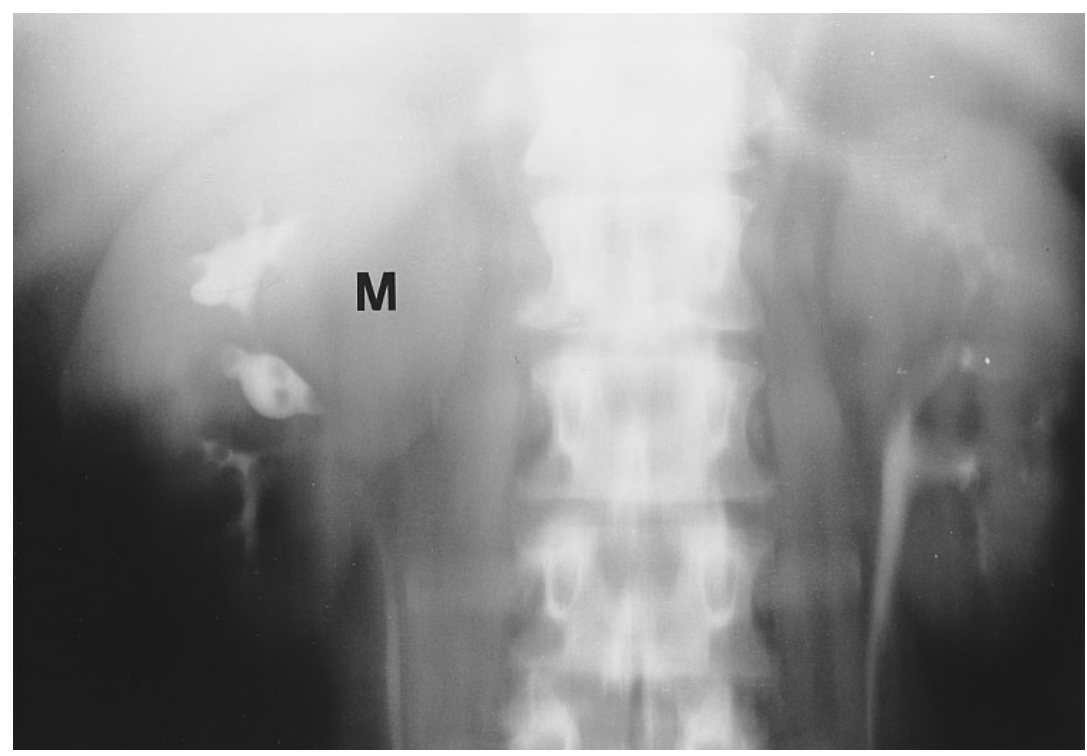

Fig. 1. Lung metastasis. A mass $(M)$ is distorting the contour of the upper pole of the right kidney on this nephrotomogram from an IVP. because they seldom erode into the collecting system. A rare case of a colon cancer metastasis presenting as a large filling defect in the renal pelvis has been reported, but it may have been introduced into the collecting system by prior percutaneous nephrostomy placement.

Ultrasound is more sensitive than excretory urography for the detection of renal metastases, detecting eight of 10 and four of six renal metastases in two recent series [7, 9]. Limitations for detection by ultrasound include the small size of the mass $(1-2 \mathrm{~cm})$, an echotexture indistinguishable from that of normal renal parenchyma, and poor visualization of the kidneys. The sonographic appearance of a metastasis is a homogenous hypoechoic mass without increased through transmission [9]. However, some metastases may appear heterogeneous or echogenic [7]. Ultrasound findings correlate well with CT findings, but the sonogram may underestimate the number of lesions because some are too small to detect [7].

The modality most frequently used to detect renal metastases is CT. Not only is CT the most sensitive modality for the detection of renal metastases, but CT also can evaluate the extent of disease and demonstrate other sites of involvement (Fig. 2). Antemortem diagnoses of renal metastases are made routinely in the absence of any renal symptomatology because cancer patients frequently undergo staging CT examinations [7, 10]. Metastatic renal tumors are typically multifocal, contained within the renal margin, and either isodense or slightly low (10-40 Hounsfield units [HU]) in attenuation relative to renal parenchyma on unenhanced CT examinations [7-9]. Small lesions that do not deform the renal contour or collecting system would be nonapparent on a noncontrast study. Typically, these small tumors are hypovascular and enhance only slightly $(5-15 \mathrm{HU})$ after intravenous contrast administration.

The CT appearance of metastases is nonspecific. They may present as multiple, bilateral, small, low-attenuation lesions on contrast-enhanced scans and may simulate renal cysts (Fig. 3). Careful scrutiny of the enhanced CT images should allow distinction of a metastasis from a cyst because the metastasis has an irregularly thickened wall and a blurred margin with the normal renal parenchyma. Furthermore, there is enhancement of the soft tissue components of the metastasis but no enhancement of a benign cyst.

A small percentage of primary renal adenocarcinomas are small, homogeneous, hypovascular masses that are indistinguishable from metastasis [11]. In general, however, renal adenocarcinomas are large, exophytic, hypervascular tumors that are inhomogeneous and demonstrate areas of necrosis. They may have associated lymph node enlargement and tumor thrombus extending into the renal vein or inferior vena cava.

Although autopsy data suggest that primary renal neoplasms are only half as prevalent as metastases, these data include microscopic metastasis below the size threshold of CT [11]. The likely diagnosis of a renal mass in a patient with a known extrarenal primary neoplasm depends on the clinical situation. For example, known malignant disease in remission favors renal adenocarcinoma, whereas widespread metastatic disease favors a new metastasis. The likelihood of a primary renal carcinoma is increased if the renal mass is discovered in an asymptomatic patient. In addition, there are rare reported cases of metastases from extrarenal neoplasms, usually lung cancer, to a primary renal adenocarcinoma [12]. This result may occur in up to $15 \%$ of patients with renal adenocarcinoma and a second primary neoplasm. 

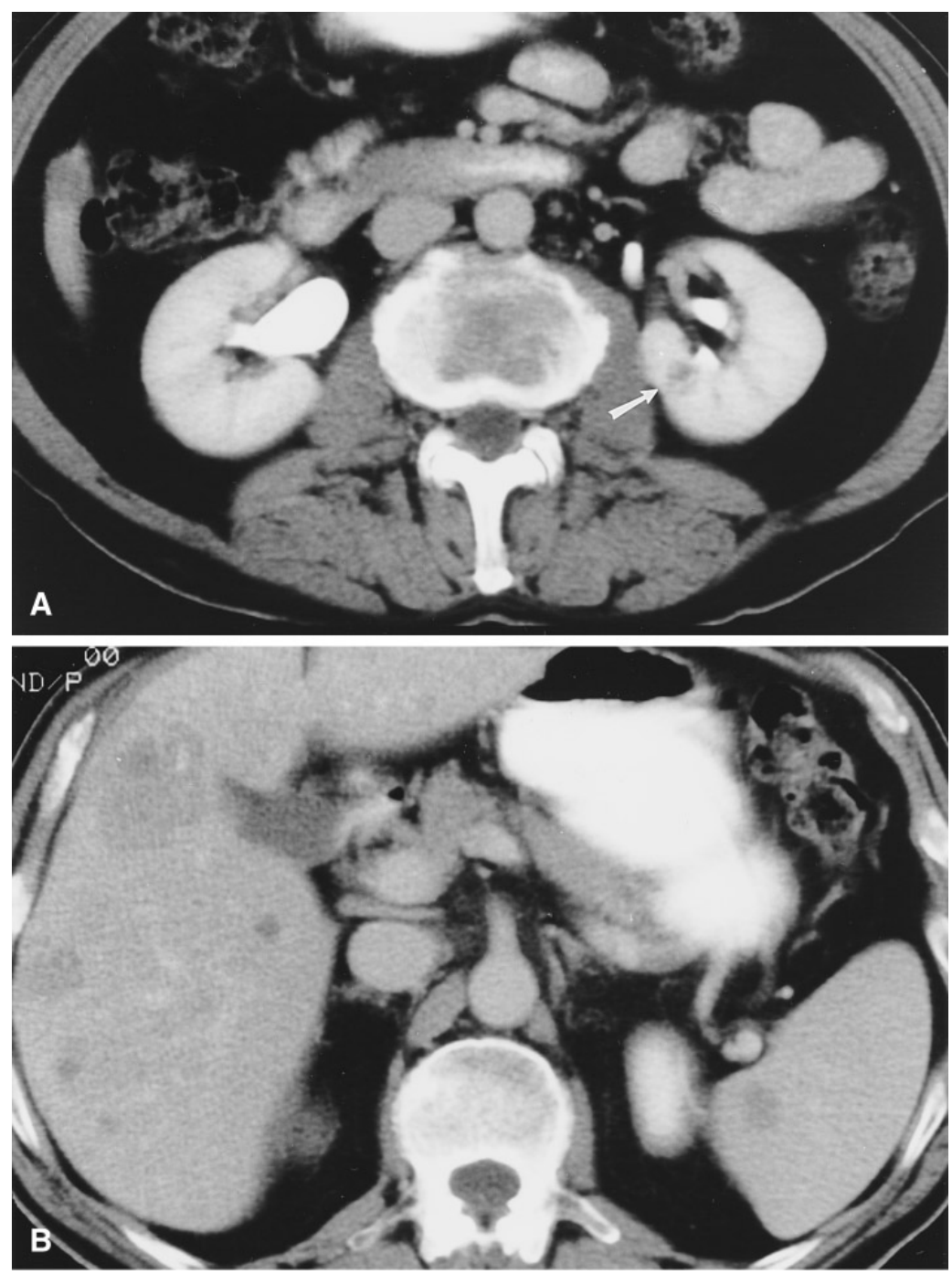

Fig. 2. Melanoma metastases. A A small heterogenous mass (arrow) is seen in the left kidney and right perinephric space on this enhanced CT image. B Multiple metastases are also seen in the liver and spleen.
Angiography is no longer commonly performed for the diagnosis of renal masses, but it may be useful in selected cases. The typical angiographic appearance of a renal metastasis is a hypovascular mass without neovascularity (Fig. 4). Small vessels may be irregular or amputated with possible encasement of vessels, but there is typically no arteriovenous shunting or significant tumor blush [13]. Exceptions are highly vascular primary neoplasms such as choriocarcinoma and melanoma, which may have highly vascular metastases $[8,14]$. Angiography is seldom performed unless transcatheter embolization of the tumor is contemplated.

Benign conditions can also be confused with renal metastases. A renal abscess may be distinguished from tumor if there is associated thickening of Gerota's fascia and/or clinical signs of infection. Renal infarct can be diagnosed by its wedge shape, thin perfused rim of cortex, lack of enhancement, and progression to scar on follow-up examinations. When imaging studies fail to yield a diagnosis, percutaneous biopsy can be performed with either CT or ultrasound guidance (Fig. 5).

\section{Renal lymphoma}

Renal involvement is a common, but usually late, manifestation of lymphoma. In an autopsy series of 696 lymphoma patients, Richmond et al. found involvement of the kidneys in $13 \%$ of patients with Hodgkin disease (HD) and $47 \%$ of patients with nonHodgkin lymphoma (NHL) [15]. The disease affected both kidneys in $74 \%$ of patients, presented with multiple nodules in affected kidneys $61 \%$ of the time, and was usually found in patients with widely disseminated disease. However, only $23 \%$ of patients had antemortem clinical evidence of renal involvement, only $14 \%$ of patients' renal lymphoma was recognized before death, and in only $0.5 \%$ of cases was renal involvement thought to be the main or sole cause of 


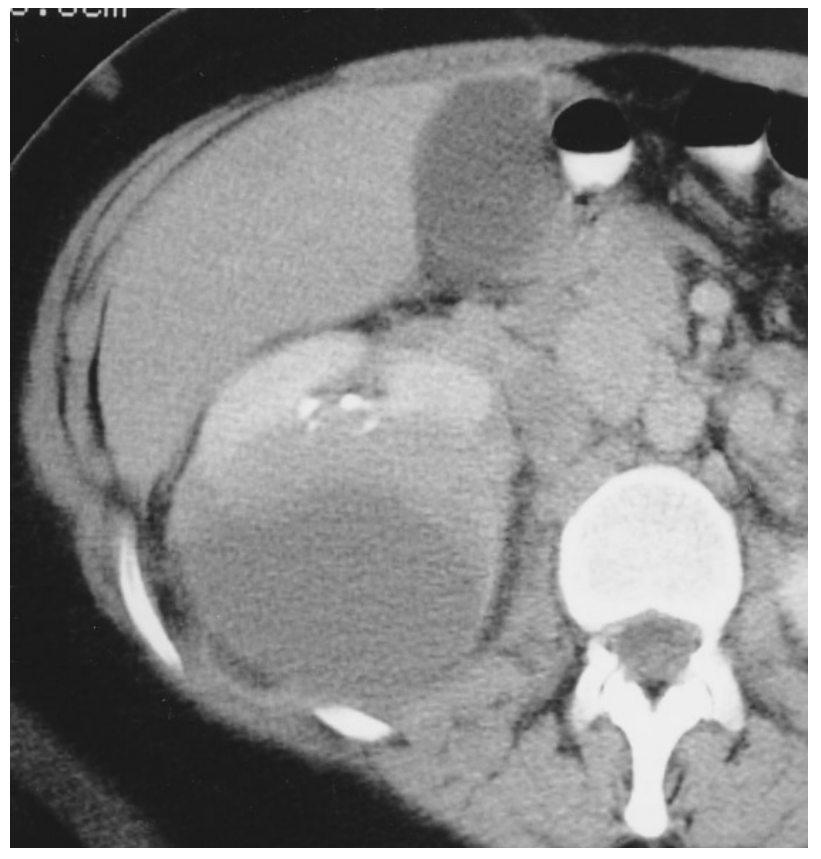

Fig. 3. Cystic metastasis. This low-density rounded mass can be distinguished from a renal cyst by the wide zone of transition from the cystic portion of the metastasis to the normal renal parenchyma.

death. When patients with widely disseminated disease were excluded, Freeman et al. found a $24 \%$ incidence of extranodal involvement but only a $0.7 \%$ incidence of renal disease [16]. Similarly, in a series of 423 patients with NHL evaluated at the time of presentation, $31 \%$ had extranodal sites of lymphoma, but only $0.5 \%$ were found to have renal disease. Most of the extranodal disease involved the liver, skin, lung, small bowel, and stomach [17].

Because renal parenchyma is not destroyed until lymphomatous invasion is extensive, renal involvement is usually clinically silent. Lymphoma cells, which tend to be diffuse large cell types when involving the kidney, may enter the kidney via hematogenous spread or via direct extension through the renal capsule $[18,19,21]$. Once in the kidney, tumor cells initially proliferate along an interstitial scaffolding made of nephrons, collecting ducts, and vessels, spreading the nephrons apart but not interfering with their function [20]. The contour of the kidney is maintained, and the edges of the mass are irregular. Often multiple small nodules coalesce to give the appearance of diffuse involvement. If the tumor becomes large enough, it will destroy the underlying renal architecture, expand the kidney, and surround the collecting system, eventually destroying all parenchyma. If growth is very rapid, the mass can project outside the renal contour and displace the collecting system. The infiltrative pattern makes lymphoma poorly marginated and unencapsulated, help-

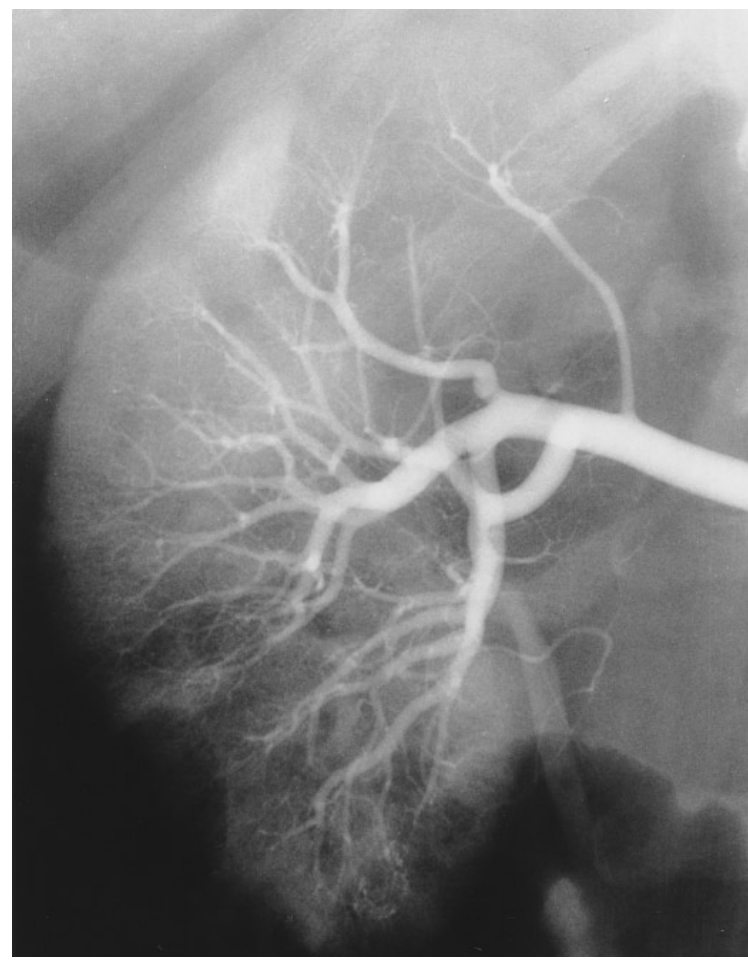

Fig. 4. Lung metastasis. A hypovascular mass is present in the right upper pole (same patient as shown in Fig. 1).

ing to distinguish it from a renal adenocarcinoma in this setting. Involvement of the perirenal space with spread of tumor into the renal sinus is not uncommon [18-20].

\section{Imaging of renal lymphoma}

Renal lymphoma lesions are often too small to be appreciated on gross pathology or on excretory urography [22]. The findings of diffuse renal enlargement, a large renal or perinephric mass, a nonfunctioning kidney, hydronephrosis, or distortion of the collecting system are due to extensive disease [20].

Sonography demonstrates hypoechoic renal nodules that have a poorly defined distal wall and no increased through transmission. The kidneys may be enlarged, and there may be hydronephrosis [20, 23]. After chemotherapy, renal lymphoma can become more heterogeneous [23]. If there is involvement of the renal sinus, the sinus fat may be infiltrated, simulating hydronephrosis or a renal pelvis tumor such as transitional cell carcinoma (Figs. 6, 7). Extensive perinephric involvement appears as thick hypoechoic tissue that may completely surround one or both kidneys [24-26]. This extensive perinephric involvement tends to occur in stage IV disease [24]. The differential diagnosis for hypoechoic perinephric soft tissue includes hematoma, uri- 

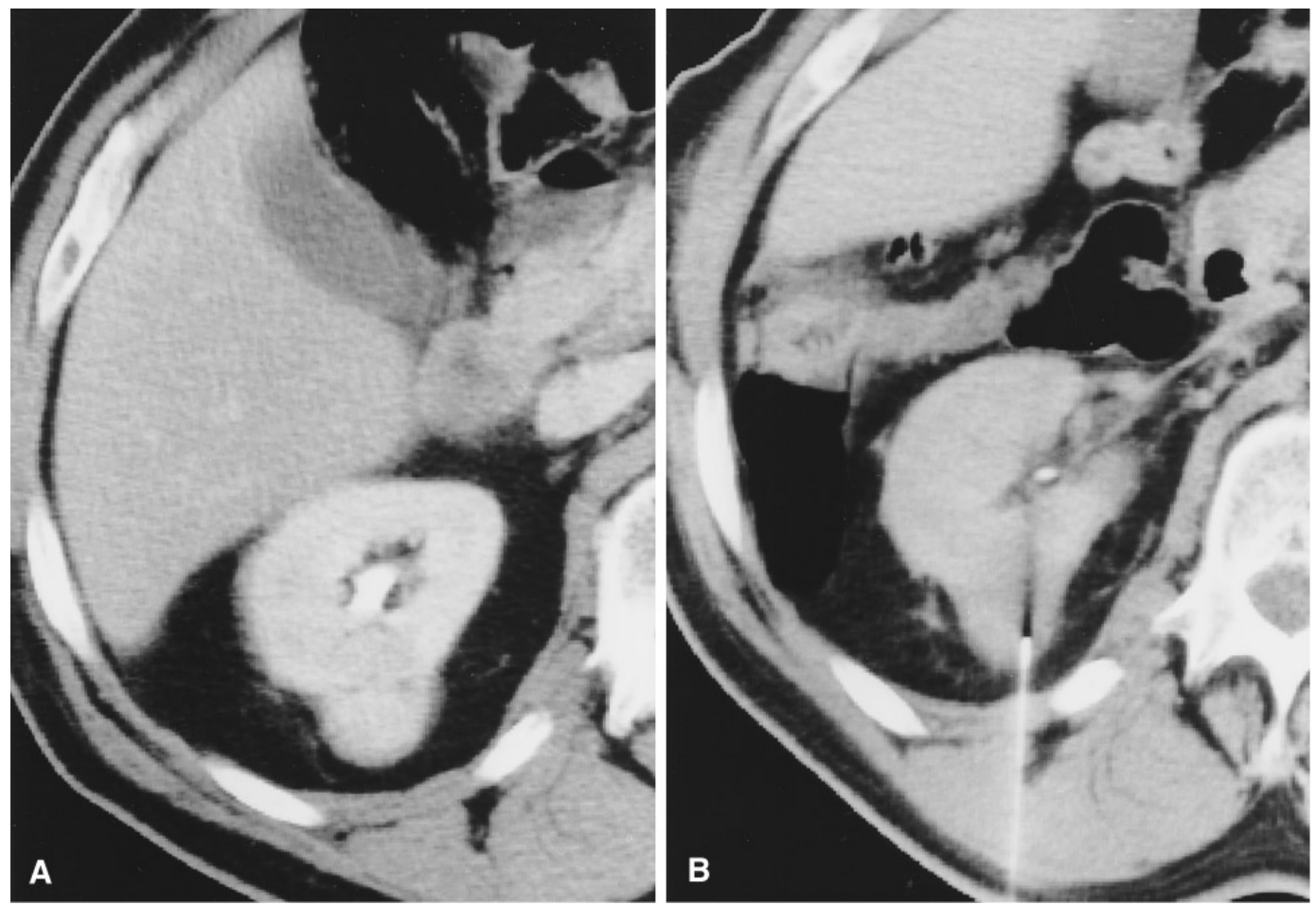

Fig. 5. Renal mass biopsy. A A homogeneous peripheral mass is detected on enhanced CT in this 70-year-old man with small cell car-

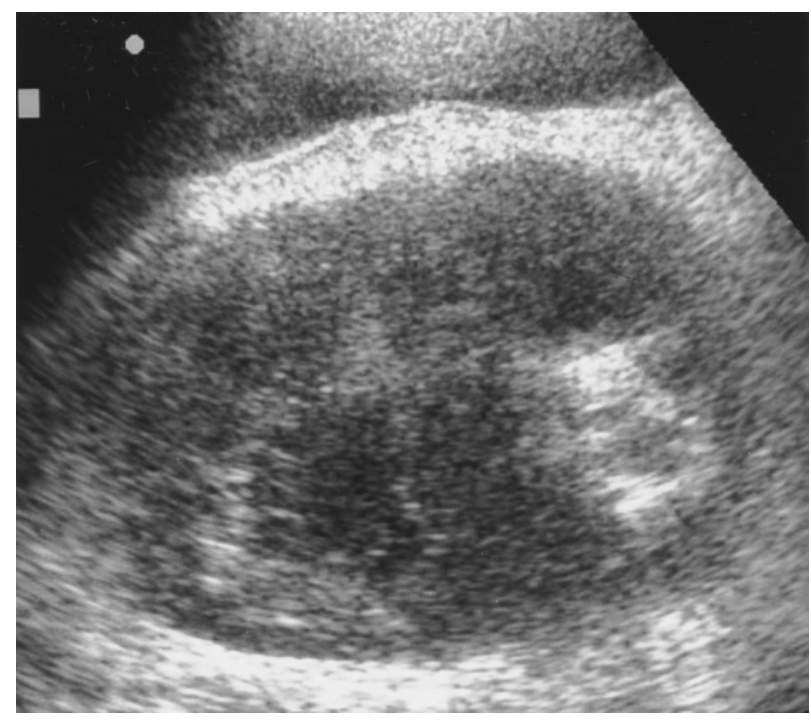

Fig. 6. Renal lymphoma. Sonogram demonstrates echogenic renal sinus fat replaced by hypoechoic lymphoma.

noma, abscess, and inflammation of Gerota's fascia [26].

CT is the preferred modality for the evaluation of renal lymphoma. When the history of lymphoma is known or when retroperitoneal lymphadenopathy is seen along with the renal findings, the CT diagnosis of cinoma of the lung. B Percutaneous biopsy under CT guidance revealed a metastasis.

renal lymphoma is not difficult. The rare renal lymphoma with associated renal vein and IVC thrombosis will lead to misdiagnosis as renal adenocarcinoma in some cases [27, 28]. Several typical CT patterns of involvement have been described.

\section{Multiple renal masses}

The most common appearance of renal lymphoma is multiple renal masses. This pattern is observed in more than $50 \%$ of patients in recent reported series, and involvement is often bilateral [18, 23, 29]. The masses have a soft tissue density, but enhance much less than normal renal parenchyma after intravenous contrast administration (Fig. 8). They are homogeneous before contrast, minimally heterogeneous after contrast, and generally cause minimal or no deformity of the renal contour. Although most series have reported associated retroperitoneal lymph node enlargement (Fig. 8), Cohan et al. found no associated retroperitoneal lymphadenopathy in 10 of 17 patients and in four of the remaining seven patients, lymph node involvement was subtle (Fig. 9) [18]. This observation was corroborated by Reznek et al. who reported coexisting retroperitoneal lymphoma in only $57 \%$ of patients [19].

\section{Invasion from contiguous retroperitoneal masses}

This pattern is seen in approximately $25 \%$ of patients. Typically there is invasion of the renal hilum, renal si- 

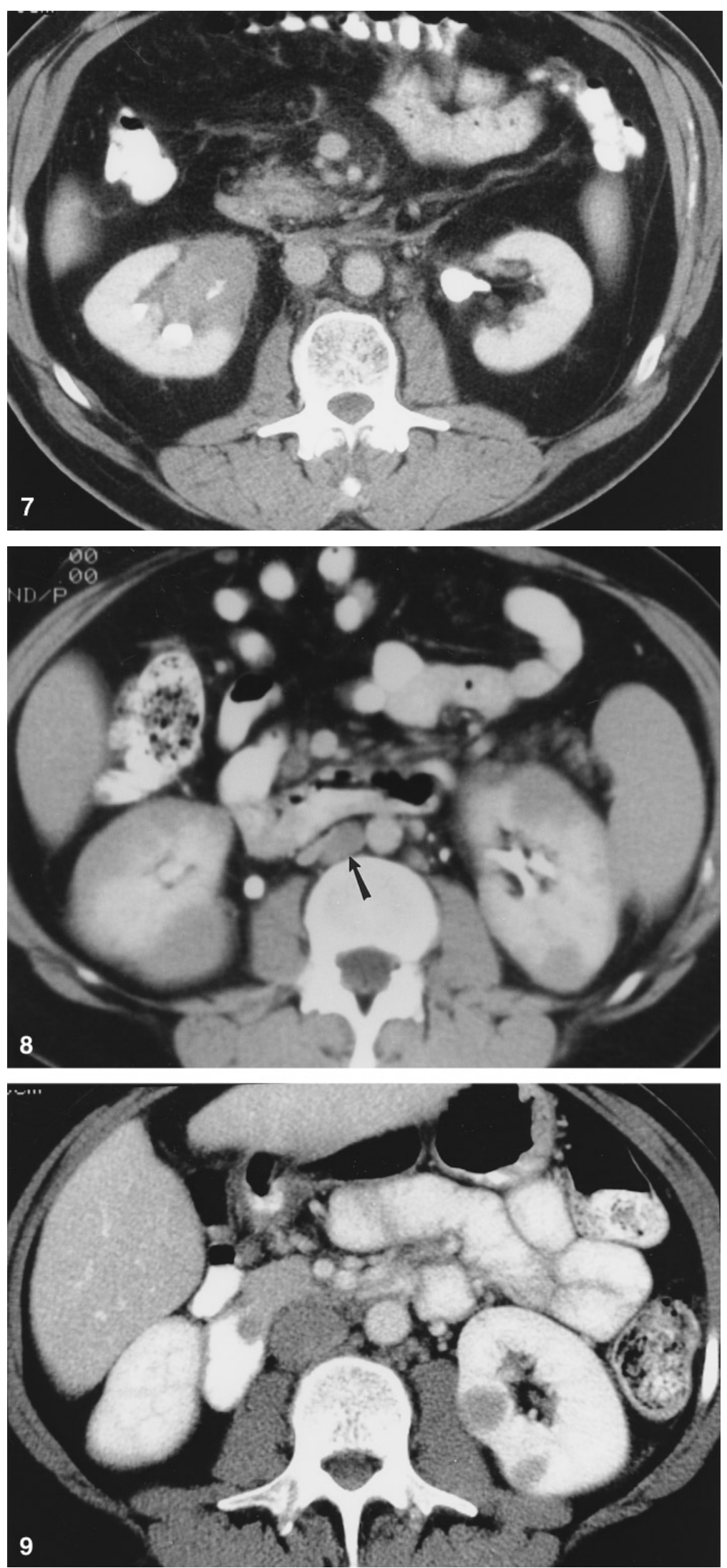

Fig. 7. Lymphoma completely replaces the renal sinus fat in the right kidney, without disturbing the normal renal contour.

Fig. 8. Renal lymphoma. Multiple renal masses are visible in the periphery of both kidneys on enhanced CT. Note the enlarged aortocaval lymph node ( $a r$ row), suggesting involvement.

Fig. 9. Renal lymphoma. Involvement of the left kidney is easily seen on enhanced CT. However, multiple retroperitoneal lymph nodes are each within normal limits for size. 

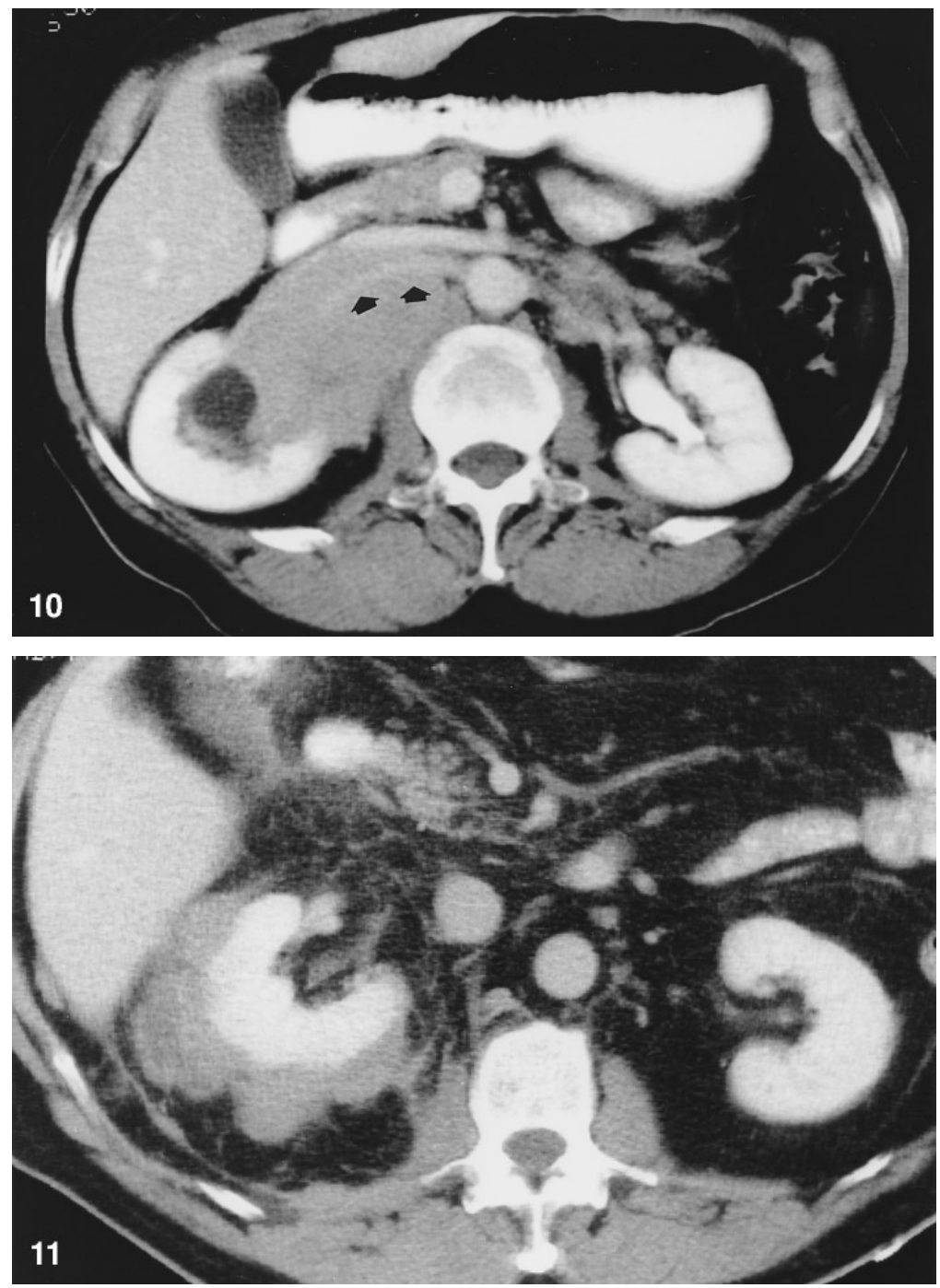

Fig. 10. Renal lymphoma. Retroperitoneal lymphoma is growing into the right kidney. Note encasement of the right renal artery (arrowheads).

Fig. 11. Perinephric lymphoma. A mass of lymphoma is seen in the right perinephric space on contrast-enhanced CT. There is stranding and thickening of Gerota's fascia. nus, or kidney by a usually homogeneous retroperitoneal lymph node mass that frequently encases the renal vessels (Fig. 10). Most patients in this category have bulky disease elsewhere in the abdomen.

\section{Solitary renal mass}

This unusual pattern is seen in only $5-15 \%$ of kidneys in renal lymphoma patients and is found even less frequently in the most recent reports $[18,23]$. Improvements in cross-sectional imaging techniques allow for detection of smaller lesions than was possible previously. Thus, renal lymphoma detected as a solitary mass may now be recognized as multiple nodules. Associated retroperitoneal lymphadenopathy or involvement of abdominal viscera is usually present.

\section{Perinephric masses}

In approximately $10 \%$ of patients, perinephric masses make up the bulk of the disease without significant in- filtration of the kidney. More commonly seen is thickening of Gerota's fascia or stranding in the perinephric fat, with the bulk of tumor in the kidney itself (Fig. 11).

Angiography has been largely replaced by CT for the diagnosis of renal masses. Angiographic findings associated with renal lymphoma are wide displacement of arteries arranged in an orderly fanlike pattern (palisade) throughout the mass or entire kidney, attenuated irregular vessels, and no tumor blush or arteriovenous shunting [30, 31].

The use of MR imaging for the evaluation of lymphoma has not been clearly established [32]. MR imaging may be useful to evaluate bone marrow disease in leukemia and in lymphoma. Bone marrow aspirates, even if obtained from two sites, may not detect disease on occasion due to sampling error. Because MR imaging can evaluate a greater volume of marrow, it may help by identifying the optimal site for bone marrow aspiration [33]. 


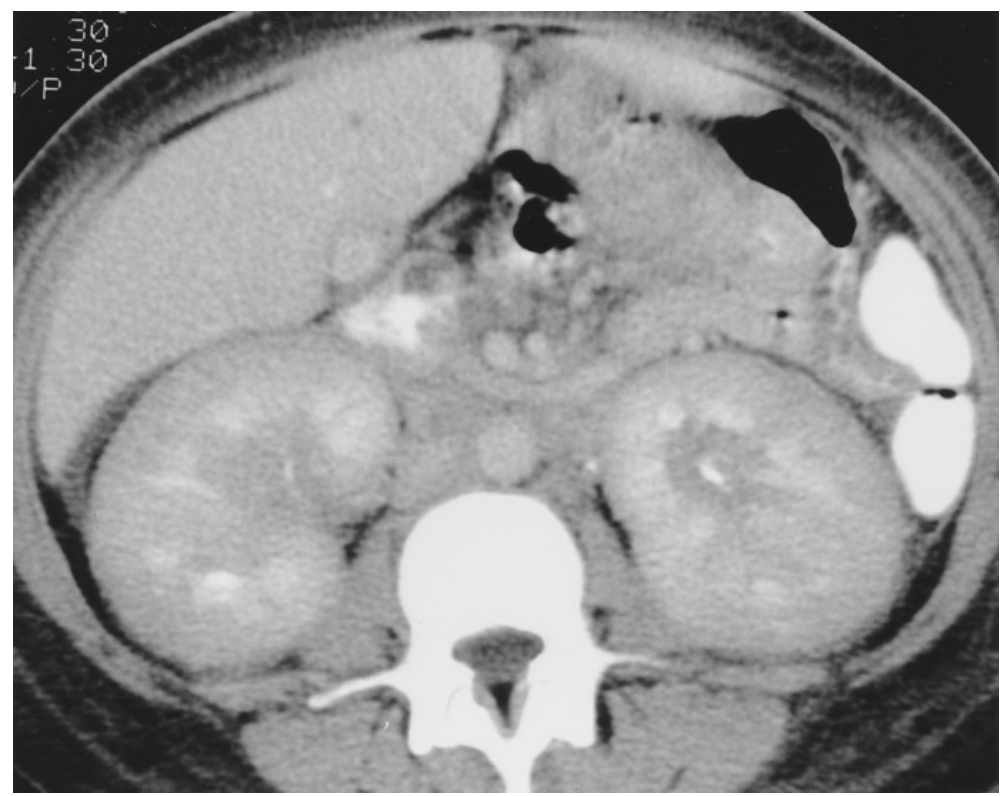

Fig. 12. Renal leukemia. Diffusely enlarged kidneys are demonstrated on contrast-enhanced CT.
Burkitt Lymphoma involves the kidneys in about $5 \%$ of cases, with radiographic findings similar to those seen in patients with NHL. More commonly, extranodal disease is manifest by small bowel wall thickening, ascites, and pleural effusions [34].

Primary renal lymphoma is rare. Because lymphoid tissue does not normally reside in the kidney, renal involvement by lymphoma is almost always secondary. On occasion, the kidney will be the first site of lymphomatous involvement, but disseminated disease usually soon becomes apparent. Metastatic disease is overwhelmingly the most common etiology of renal lymphoma, but there have been isolated reported cases of renal lymphoma involving only one kidney and never recurring after nephrectomy $[21,35-37]$. One theory as to the origin of primary renal lymphoma suggests that inflammation in the kidney attracts lymph cells that at some point transform into malignant lymphoma while in the kidney [36].

Lymphoma is a well-known complication of AIDS, primary immunodeficiency disorders, and iatrogenic immunosuppression in transplant recipients. This patient population has a risk for developing lymphoma that is $30-70$ times the rate of the normal population [38]. The majority of these tumors are B-cell origin, high-grade, small, noncleaved cell lymphomas. Extranodal disease is extremely common, especially involving the central nervous system. Of the extranodal sites not in the central nervous system, the lung and genitourinary tract are the most frequently involved [39]. As the number of AIDS cases continues to increase, the number of renal lymphomas will likely increase [40].

\section{Renal leukemia}

Renal leukemia is primarily a disease of children, especially patients with lymphocytic leukemia, which has a higher predilection for renal involvement than the myelogenous leukemias. Pathologically, leukemic infiltration tends to occur interstitially, infiltrating between nephrons and globally enlarging the kidney in some instances. Patients with leukemic infiltration of the kidneys are usually asymptomatic [41].

Fifty-two percent of end-stage leukemia patients have leukemic infiltration of the kidneys [41]. The most common clinical and imaging finding is diffuse bilateral renal enlargement, with or without other organomegaly [42]. Organomegaly is not, however, diagnostic of leukemic infiltration. Although two-thirds of patients autopsied after dying of leukemia have organomegaly, in only one-half of these cases is the organomegaly due to leukemic infiltration [43]. Other causes include parenchymal hemorrhage, often due to profound thrombocytopenia with generalized hemorrhagic manifestations, and infection [41].

\section{Imaging of renal leukemia}

Excretory urography demonstrates bilateral symmetrically enlarged kidneys with smooth renal contours. Unilateral enlargement is occasionally seen. Leukemic infiltration of the kidneys rarely leads to renal failure, but urate nephropathy may result from the very high cellular turnover, leading to nonvisualization on urography.

Sonographic findings are similar, demonstrating bilateral enlarged hypoechoic kidneys. The differential diagnosis for nephromegaly in this patient population includes leukemic infiltration, hemorrhage, infection, edema, necrosis, and cellular hypertrophy [42]. When nephromegaly is part of panorganomegaly, the chance of leukemic infiltration is high. If, however, nephro- 
megaly is an isolated finding, its correlation with hematologically active disease is low [42].

CT is not regularly performed in this patient population but some patterns have been described. Diffuse parenchymal thickening is most common, usually involving both kidneys (Fig. 12). Focal renal masses and masses at the renal hilum can also be seen [44]. Hematoma is the most common complication. These occur most often into large muscles such as the psoas or iliopsoas and are readily detected by CT [45].

\section{References}

1. Dunnick NR, Sandler CM, Amis ES, et al. Textbook of uroradiology, 2nd ed. Baltimore: Williams \& Wilkins, 1997

2. Hietala SO, Wahlqvist L. Metastatic tumors to the kidney. Acta Radiol Diagn 1982;23:585-591

3. Abrams HL, Spiro R, Goldstein N. Metastases in carcinoma. Cancer 1950;3:74-85

4. Klinger ME. Secondary tumors of the genitourinary tract. J Urol 1951;65:144-153

5. Wagle DG, Moore RH, Murphy GP. Secondary carcinomas of the kidney. J Urol 1975;114:30-32

6. Olsson CA, Moyer JD, Laferte RO. Pulmonary cancer metastatic to the kidney-A common renal neoplasm. J Urol 1971;105:452-456

7. Choyke PL, White EM, Zeman RK, et al. Renal metastases: clinicopathologic and radiologic correlation. Radiology 1987; $162: 359-363$

8. Bhatt GM, Bernardino ME, Graham SD. CT diagnosis of renal metastasis. J Comput Assist Tomogr 1983;7:1032-1034

9. Mitnick JS, Bosniak MA, Rothberg M, et al. Metastatic neoplasm to the kidney studied by computed tomography and sonography. J Comput Assist Tomogr 1985;9:43-49

10. Thomas JL, Barnes PA, Bernardino ME, et al. Diagnostic approaches to adrenal and renal metastases. Radiol Clin North Am 1982;20:531-544

11. Pagani JJ. Solid renal mass in the cancer patient: second primary renal cell carcinoma versus renal metastasis. J Comput Assist Tomogr 1983;7:444-448

12. Sella A, Ro JY. Renal cell cancer: best recipient of tumor-totumor metastasis. Urology 1987;30:35-38

13. Bosniak MA, Stern W, Lopez F, et al. Metastatic neoplasm to the kidney. Radiology 1969;92:989-993

14. Kutcher R, Lu T, Gordon DH, et al. Renal choriocarcinoma metastasis: a vascular lesion. AJR 1977;128:1046-1048

15. Richmond J, Sherman RS, Diamond HD, et al. Renal lesions associated with malignant lymphomas. Am J Med 1962;32:184-207

16. Freeman C, Berg JW, Cutler SJ. Occurrence and prognosis of extranodal lymphomas. Cancer 1972;29:252-260

17. Goffinet DR, Warnke R, Dunnick NR, et al. Clinical and surgical (laparotomy) evaluation of patients with non-Hodgkin's lymphoma. Cancer Treat Rep 1977;61:981-992

18. Cohan RH, Dunnick NR, Leder RA, et al. Computed tomography of renal lymphoma. J Comput Assist Tomogr 1990;14:933-938

19. Reznek RH, Mootoosamy I, Webb JAW, et al. CT in renal and perirenal lymphoma: a further look. Clin Radiol 1990;42:233238
20. Hartman DS, Davis CJ, Goldman SM, et al. Renal lymphoma: radiologic-pathologic correlation of 21 cases. Radiology 1982;144:759-766

21. Ferry JA, Harris NL, Papanicolaou N, et al. Lymphoma of the kidney. Am J Surg Pathol 1995;19:134-144

22. Lalli AF. Lymphoma and the urinary tract. Radiology 1969;93:1051-1054

23. Heiken JP, Gold RP, Schnur MJ, et al. Computed tomography of renal lymphoma with ultrasound correlation. J Comput Assist Tomogr 1983;7:245-250

24. Seltzer SE, Jochelson M, Balikian JP. Organ envelopment in lymphoma visualised by computed tomography. Clin Radiol 1986;37:525-529

25. Gorg C, Weide R, Schwerk WB. Unusual perirenal sonographic pattern in malignant lymphoma of the kidney. Clin Radiol 1995;50:720-724

26. Villalon FC, Fernandez JE, Garcia TR. The hypoechoic halo: a finding in renal lymphoma. J Clin Ultrasound 1995;23:379-381

27. Eyre RC, Huberman MS, Balogh K. Non-Hodgkin's lymphoma of the kidney with inferior vena caval extension. Urol Int 1993;51:43-45

28. Wagner JR, Honig SC, Siroky MB. Non-Hodgkin's lymphoma can mimic renal adenocarcinoma with inferior vena caval involvement. Urology 1993;42:720-724

29. Chilcote WA, Borkowski GP. Computed tomography in renal lymphoma. J Comput Assist Tomogr 1983;7:439-443

30. Kwaw M, Koehler PR. Renal and perirenal lymphoma: arteriographic findings. Radiology 1969;93:1055-1058

31. Pick RA, Castellino RA, Seltzer RA. Arteriographic findings in renal lymphoma. Am J Roentgenol Rad Ther Nucl Med 1971;111:530-534

32. Semelka RC, Kelekis NL, Burdeny DA, et al. Renal lymphoma: demonstration by MR imaging. AJR 1996;166:823-827

33. Shields AF, Porter BA, Churchley S, et al. The detection of bone marrow involvement by lymphoma using magnetic resonance imaging. J Clin Oncol 1987;5:225-230

34. Dunnick NR, Reaman GH, Head GL, et al. Radiographic manifestations of Burkitt's lymphoma in American patients. AJR 1979;132:1-6

35. Dimopoulos MA, Moulopoulos LA, Costantinides C, et al. Primary renal lymphoma: a clinical and radiological study. J Urol 1996;155:1865-1867

36. Kandel LB, McCullough DL, Harison LH, et al. Primary renal lymphoma Does it exist? Cancer 1987;60:386-391

37. Harris GJ, Lager DJ. Primary renal lymphoma. J Surg Oncol 1991;46:273-277

38. Egerter DA, Beckstead JH. Malignant lymphomas in the acquired immunodeficiency syndrome. Arch Pathol Lab Med 1988;112:602-606

39. Loureiro C, Gill PS, Meyer PR, et al. Autopsy findings in AIDSrelated lymphoma. Cancer 1988;62:735-739

40. Townsend RR. CT of AIDS-related lymphoma. AJR 1991;156: 969-974

41. Norris HJ, Wiener J. The renal lesions in leukemia. Am J Med Sci $1961 ; 241: 512-518$

42. Gore RM, Shkolnik A. Abdominal manifestalons of pediatric leukemias: sonographic assessment. Radiology 1982;143:207-210

43. Frei E, Fritz RD, Price E, et al. Renal and hepatic enlargement in acute leukemia. Cancer 1963;16:1089-1092

44. Araki T. Leukemic involvement of the kidney in children: CT features. J Comput Assist Tomogr 1982;6:781-784

45. Heiberg E, Wolverson MK, Sundaram M, et al. CT findings in leukemia. AJR 1984;143:1317-1323 